\title{
ENSAIO RADIOGRÁFICO DE JUNTAS LAMINADAS DE MATERIAL POLIMÉRICO REFORÇADO POR FIBRA DE VIDRO*
}

\author{
Cintia Guimarães Ferreira ${ }^{1}$ \\ Davi Ferreira de Oliveira ${ }^{1}$ \\ Ricardo Tadeu Lopes ${ }^{1}$ \\ Fabiana Dias Fonseca Martins² \\ Gabriela Ribeiro Pereira ${ }^{3}$
}

\section{Resumo}

O uso de materiais compósitos possibilitou relevante avanço tecnológico em áreas biomédicas e industriais. As matrizes poliméricas reforçadas por Fibra de Vidro são predominantes em nível comercial devido à facilidade no processo de fabricação. Entretanto, podem apresentar variabilidade intrínseca de propriedades devido a fatores importantes, tais como: não uniformidade e não conformidades relativas ao reforço e matriz. Inspeções visuais identificam defeitos característicos de distorção de fibras, bolhas superficiais e áreas secas de impregnação. Para detectar porosidades, trincas ou delaminações outros métodos são necessários. Este estudo apresenta a técnica de Radiografia Direta (RD) para a inspeção de materiais compósitos. Utilizouse um detector Flat Panel do tipo silício amorfo ( $\alpha \mathrm{Si}$ ), uma fonte de raios $\mathrm{X}$ de potencial constante e uma mesa controlada por Arduino. Foram realizadas radiografias de duas juntas de 4 polegadas de diâmetro nominal de material polimérico reforçado por fibra de vidro. Como resultado, foram detectados defeitos de delaminação, descolamento, trincas e porosidades, que não poderiam ser detectados visualmente.

Palavras-chave: Radiografia Digital; Materiais Compósitos; Fibras.

\section{RADIOGRAPHIC ASSAY OF LAMINATED JOINTS OF POLYMERIC MATERIAL REINFORCED BY GLASS FIBER}

\section{Abstract}

The use of composite materials made possible technological advances in biomedical and industrial areas. Polymeric matrices reinforced by Glass Fiber are predominant with respect to manufacturing. However, they may present intrinsic properties variability due to important factors, such as: non-uniformity and non-conformities related to reinforcement and matrix. Visual inspections identify defects characteristic of fiber distortion, surface bubbles, and dry areas of impregnation. To detect porosity, cracking or delamination other methods are required. This study presents the technique of Direct Radiography (RD) for the inspection of composite materials. An amorphous silicon-type Flat Panel detector ( $\alpha \mathrm{Si})$, a constant potential X-ray source, and an Arduino-controlled table were used. Radiographs of two 4-inch diameter nominal joints of glass fiber reinforced polymer material were performed. As a result, delamination, detachment, cracking and porosity defects were detected, which could not be detected visually.

Keywords: Direct Radiography; Composite materials; Fibers.

1 Laboratório de Instrumentação Nuclear, COPPE, Universidade Federal do Rio de Janeiro, Rio de Janeiro,Brasil-cintia_gf@lin.ufr.br.

2 CENPES/Petrobrás - fabianadf@petrobras.com.br.

3 Programa de Engenharia Metalúrgica e de Materiais-COPPE/UFRJ - gpereira@metalmat.ufrj.br. 


\section{INTRODUÇÃO}

A crescente utilização de materiais compósitos em aplicações aeroespaciais, biomédicas, indústria automobilística, entre outros, exige, na mesma proporção, a busca por informações técnicas e maior aprofundamento no assunto. Combinações de diferentes materiais resultam num componente de características físicas, químicas e mecânicas únicas. E o resultado final depende, além dos constituintes e respectivas frações volumétricas, de todo o processo de fabricação adotado.

Dentre os materiais compósitos, os de matriz polimérica são mais comumente utilizados, pois se processam a temperaturas abaixo de $300^{\circ} \mathrm{C}$ e não necessitam de altas pressões de moldagem [1]. Em relação ao reforço, a fibra de vidro é o tipo mais utilizado em compósitos com matriz polimérica devido ao baixo custo e alta resistência à tração.

Em relação ao processamento de juntas laminadas, o processo de moldagem manual ( hand lay-up) não envolve nenhum investimento em equipamentos para a manufatura, sendo dependente das habilidades do operador, em quase sua totalidade. Deste tipo de processamento podem resultar compósitos sem compromisso estrutural, com uma pequena fração volumétrica de reforço e com consideráveis frações volumétricas de vazios [2].

A inspeção é uma etapa importante em um procedimento básico de avaliação da qualidade de preparação de corpos de prova para determinação de admissíveis de projeto $[3,4]$. A inspeção visual é capaz de identificar defeitos característicos de distorção de fibras, bolhas superficiais ou áreas secas. Entretanto, apresenta-se ineficiente para detectar trincas, porosidades e defeitos internos ao material. Técnicas de ultrassom podem ser utilizadas como alternativa de inspeção, mas apresentam limitações devido à heterogeneidade e anisotropia do material [5]. E justamente, essas características, tornam o uso da radiografia eficiente na inspeção de materiais compósitos.

O objetivo deste trabalho é apresentar os resultados obtidos com a técnica de Radiografia Direta na inspeção de juntas laminadas de material polimérico reforçado por fibra de vidro.

\subsection{Revisão de literatura}

A Radiografia é uma técnica de inspeção que utiliza uma fonte de raios $X$ e um detector (analógico ou digital) para obter imagens do interior de um corpo de prova sem a necessidade de destruí-lo. A técnica baseia-se na absorção diferenciada da radiação penetrante, pelo corpo de prova inspecionado, onde as diferenças na densidade, espessura e composição do material, influenciam na imagem final.

Muito utilizada para diversos tipos de aplicações, a radiografia é capaz de detectar com boa sensibilidade defeitos de pequenas espessuras, trincas e vazios internos. A técnica tem acompanhado as demandas do setor industrial apresentando evoluções consideráveis no que tange aos detectores utilizados [6].

Em sua versão mais recente, conhecida como Radiografia Direta (RD), utiliza-se detectores Flat Panel ou lineares (ou Digital Detector Array - DDA). Com este detector, exclui-se a fase de extração da imagem latente exigida nos processos analógicos, pois os resultados são obtidos em tempo real, diretamente na tela do computador [7].

O Flat Panel possui milhões de pixels atuando como detectores individuais. Trata-se de um dispositivo eletrônico, composto por um arranjo discreto de sensores 
analógicos, capaz de converter a radiação penetrante em sinais que serão digitalizados e transferidos para a tela de um computador. Detectores do tipo silício amorfo ( $\alpha \mathrm{Si}$ ) contém materiais cintiladores na estrutura do Flat Panel para intermediar a conversão radiação-sinal digital. Assim, uma conversão radiação-luz visível-sinal digital é gerada. A imagem digital que é formada, corresponde ao padrão de energia radiológica incidente sobre a superfície do dispositivo [8].

A RD apresenta diversos benefícios que justificam sua utilização para inspeção industrial. Para que uma imagem seja gerada na tela do computador o tempo de exposição necessário pode corresponder a alguns segundos ou poucos minutos, otimizando o serviço. O baixo tempo de exposição leva em consideração a curva de sensibilidade, que para o Flat Panel, apresenta-se 100 vezes mais sensível do que detectores convencionais.

Embora o Flat Panel tenha uma resolução espacial básica (SRb) limitada pelo tamanho de seu pixel, essa deficiência é compensada pela alta razão sinal ruído (SNR) alcançada [9]. Outro fator a ser considerado, é a possibilidade de realizar múltiplas operações nas imagens digitais, como por exemplo, a aplicação de filtros.

\section{MATERIAIS E MÉTODOS}

Foi utilizado um sistema de Radiografia Direta contendo: 1 tubo de raios $\mathrm{X}$ com ponto focal de $1 \mathrm{~mm}$ e potência máxima de $3600 \mathrm{~W}$ e um detector Flat Panel do tipo silício amorfo ( $\alpha \mathrm{Si}$ ) com tamanho de pixel de $200 \mu \mathrm{m}$. Os corpos de prova correspondem a duas juntas laminadas de matriz epóxi reforçadas por fibra de vidro. A junta identificada como J1, em princípio, não possui defeitos. Na junta identificada como J2 foram inseridas fitas de acetato na superfície dos dutos e entre as mantas, no intuito de simular defeitos de descolamento e delaminação, que podem ocorrer durante o processo de moldagem manual. Os parâmetros geométricos das juntas são apresentados na tabela 1.

Tabela 1. Identificação dos corpos de prova

\begin{tabular}{lccc} 
Amostra & Diâmetro $(\mathbf{m m})$ & $\begin{array}{c}\text { Espessura } \\
\text { aproximada }(\mathbf{m m})\end{array}$ & $\begin{array}{c}\text { Presença de } \\
\text { defeitos }\end{array}$ \\
\hline $\mathrm{J} 1$ & $101,6(4 ")$ & mín. 13, máx. 27 & Não \\
\hline $\mathrm{J} 2$ & $101,6(4 ")$ & mín. 10, máx. 30 & Sim \\
\hline
\end{tabular}

O processo de aquisição foi semi-automatizado, onde o detector e a mesa trabalham individualmente, comandados pelo operador. A técnica de exposição utilizada foi a de Parede Dupla Vista Dupla (PDVD), onde o feixe proveniente da fonte atravessa duas espessuras projetando duas seções do corpo de prova no detector.

Os corpos de prova foram posicionados em cima de uma mesa dotada de um sistema de rotação controlado por Arduino. Para cada amostra foram realizadas 360 radiografias, rotacionando de 1 grau. O software de aquisição, fornecido pelo fabricante do detector, controla o funcionamento do Flat Panel. Nele é possível selecionar informações como ganho, quantidade de frames e tempo de aquisição. $O$ tubo de raios $X$ possui um painel de controle de tensão e corrente.

Os parâmetros operacionais utilizados foram: tensão do tubo de $70 \mathrm{kV}$, corrente de 5 $\mathrm{mA}$, tempo de aquisição de $1 \mathrm{~s}, 5$ frames e distância fonte-detector de $1200 \mathrm{~mm}$. A cada exposição, uma projeção da amostra é gerada diretamente na tela do computador. As radiografias foram analisadas individualmente através do software 
fornecido pelo fabricante do detector. A figura 1 ilustra o arranjo experimental durante a exposição.

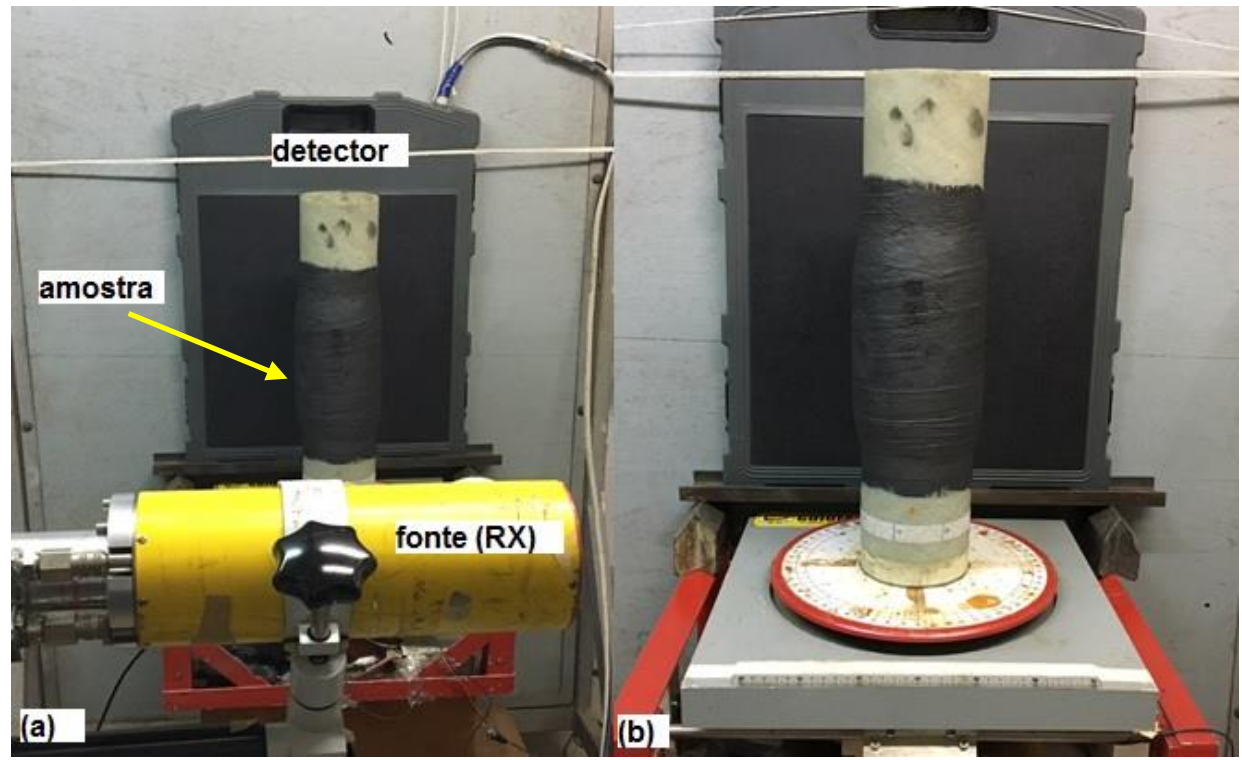

Figura 1. (a) arranjo experimental. (b) destaque para a mesa de rotação e amostra posicionada.

\section{RESULTADOS E DISCUSSÃO}

Conhecendo-se a espessura e o material do corpo de prova, os parâmetros operacionais, para obter uma radiografia, podem ser estimados teoricamente. Entretanto, o exame visual das imagens é necessário para garantir que todas as regiões de interesse estejam visíveis. As figuras 2 e 3 demonstram que os parâmetros operacionais estão adequados ao objetivo do estudo. Nas radiografias estão visíveis a união dos dutos, as soldas poliméricas e a camada laminada de cada junta.
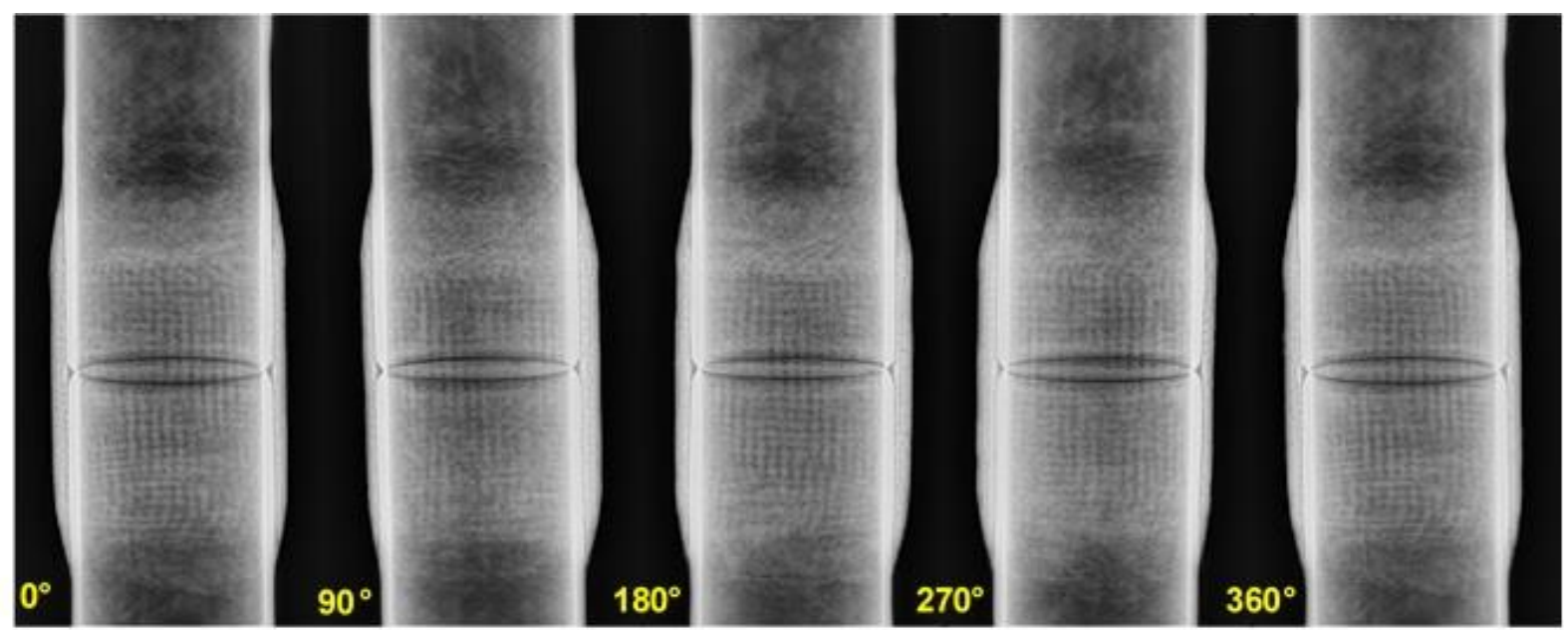

Figura 2. Radiografias da junta 1, variando o ângulo em $90^{\circ}$. 

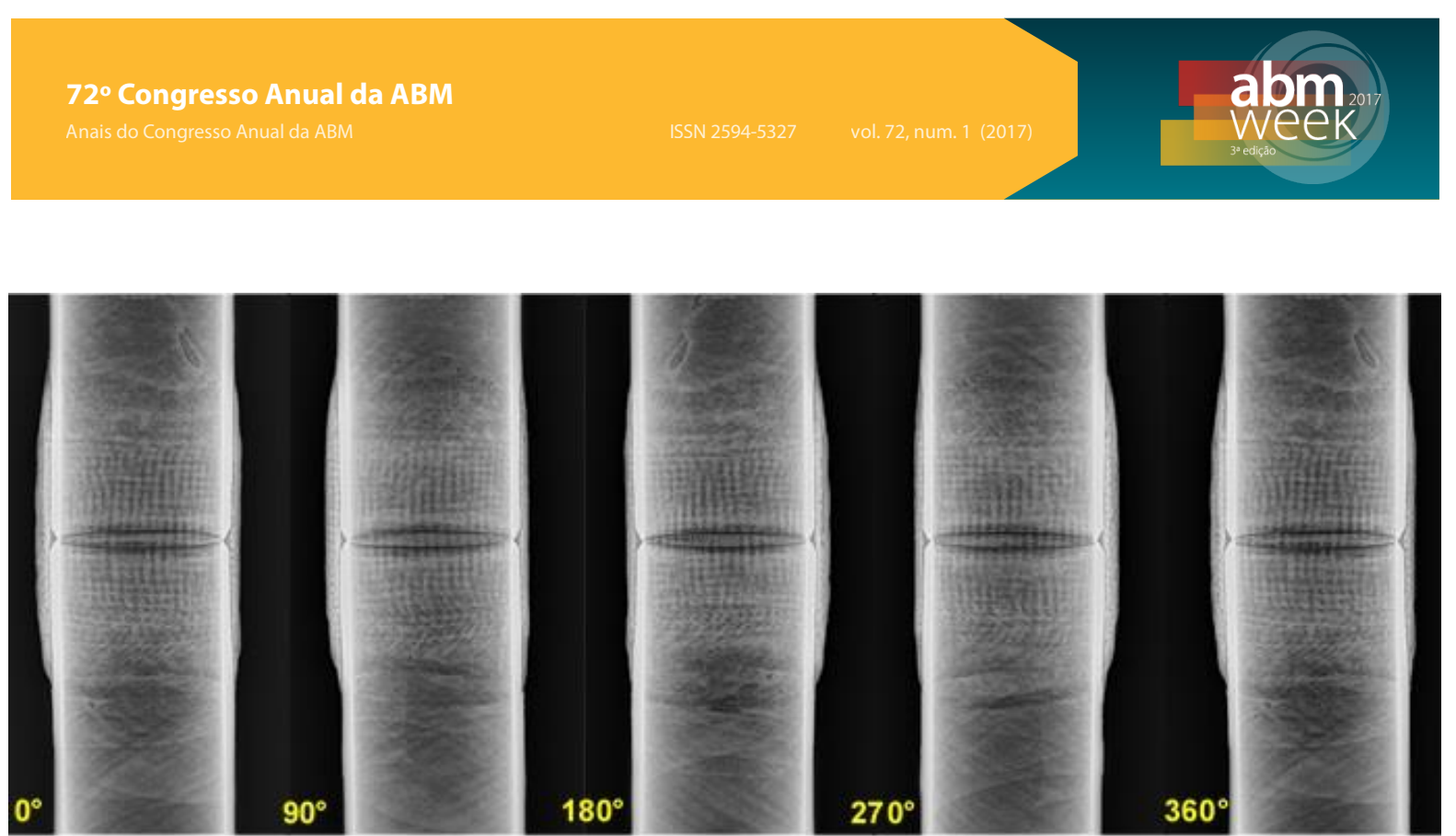

Figura 3. Radiografias da junta 2, variando o ângulo em $90^{\circ}$.

Com a variação angular foi possível observar detalhes não apresentáveis à primeira vista. As radiografias da junta 1 , entre as posições $50^{\circ}$ e $80^{\circ}$, demonstraram a presença de descolamento manta-duto. É importante ressaltar que nesta junta não foi inserido nenhum defeito proposital. Na figura 4 pode-se observar o defeito em destaque na posição $68^{\circ}$.



Figura 4. Descolamento manta-duto detectado na radiografia da junta 1.

Outro defeito detectado pelas radiografias da junta 1: a presença de trinca na parede do duto. Esse resultado foi identificado nas posições $100^{\circ}$ a $119^{\circ} \mathrm{e}$ indica que a RD é capaz de detectar pequenas alterações na densidade de materiais poliméricos. $\mathrm{Na}$ figura 5 está destacada a trinca referente a posição $106^{\circ}$. 


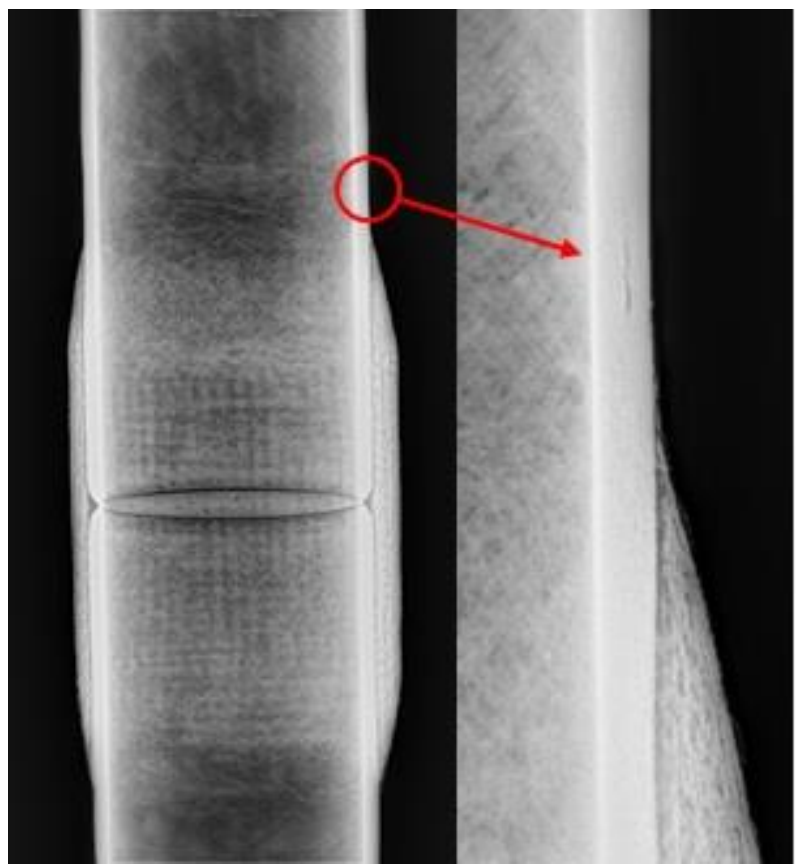

Figura 5. Trinca detectada na parede do duto da junta 1.

Conforme esperado, as radiografias da junta 2, detectaram a presença dos defeitos inseridos. Porosidades agrupadas e isoladas foram observadas em todas as posições. Além disso, um deslocamento manda-duto foi percebido em algumas posições. As figuras 6 e 7 destacam os respectivos defeitos.

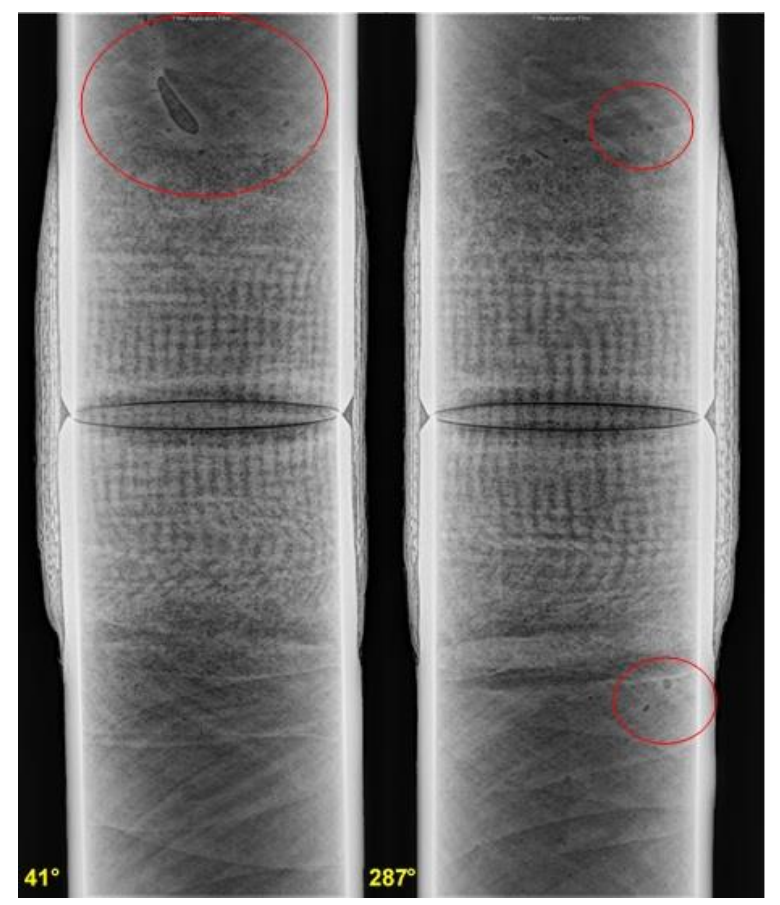

Figura 6. Presença de poros agrupados e isolados nas posições $41^{\circ}$ e $287^{\circ}$ da junta 2 . 


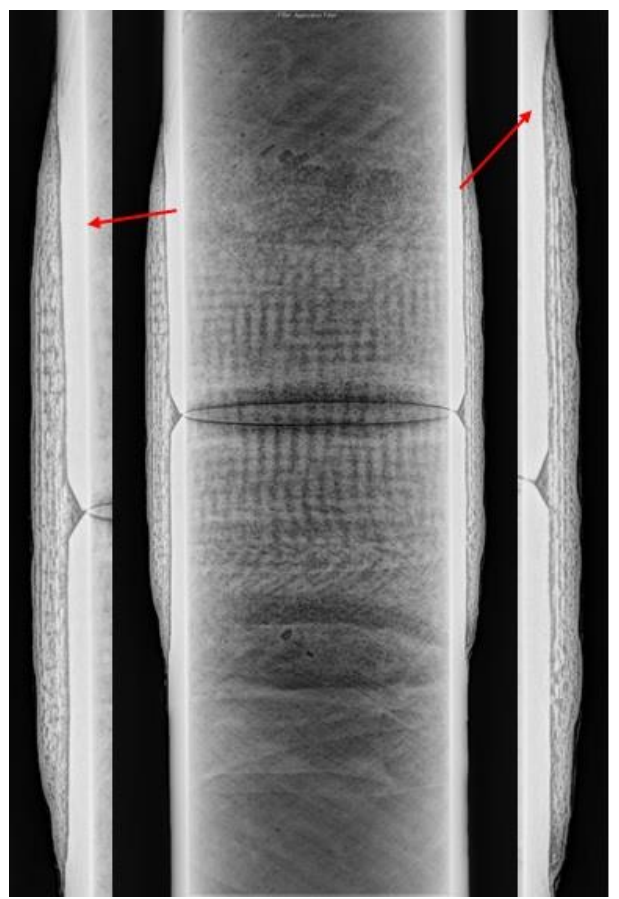

Figura 7. Descolamento verificado na posição $135^{\circ}$ da junta 2.

As fitas de acetato, inseridas entre as mantas e na superfície do duto, da junta 2 não foram bem detectadas pelas radiografias. Um padrão parece representar alguma delaminação entre as mantas, conforme demonstrado pela figura 8. Entretanto, medições mais apuradas precisam ser realizadas.

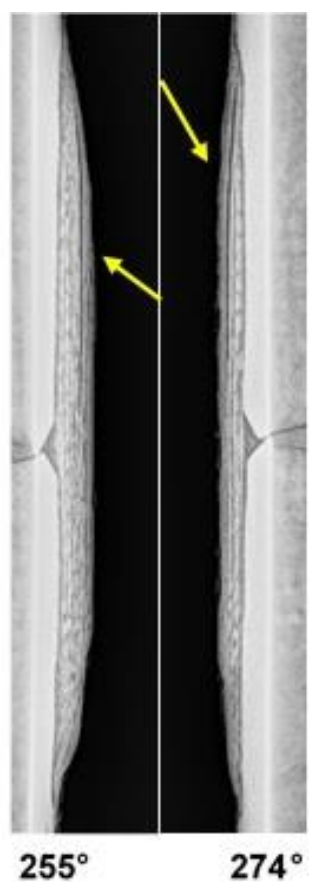

Figura 8. Padrões identificados nas posições $255^{\circ}$ e $274^{\circ}$ da junta 2. 


\section{CONCLUSÃO}

Os raios $X$ não são absorvidos da mesma forma por diferentes materiais. Por este motivo, a heterogeneidade e anisotropia dos materiais compósitos indicam bons resultados para a utilização de Radiografia Direta na inspeção de juntas laminadas de materiais poliméricos reforçados por fibra de vidro.

As radiografias demonstraram potencial para identificar os padrões de materiais presentes em ambas as juntas. A sensibilidade radiográfica permitiu detectar defeitos não previstos na junta 1 , como descolamento manta-duto e trinca. Além disso, foi capaz de detectar aqueles já previstos na junta 2. No caso das delaminações, um padrão radiográfico ficou aparente em algumas posições. Outras medições serão feitas no intuito de verificar a detecção deste tipo de defeito.

É necessário refinar as medições para que um levantamento quantitativo dos defeitos possa ser realizado. A melhoria na calibração do detector Flat Panel ou a redução do passo angular podem contribuir para a redução do ruído nas radiografias. Entretanto, para uma aplicação em campo estima-se a redução do tempo (processo completo) com o aumento do passo angular.

Há uma certa resistência em se utilizar radiografia para inspeção em campo, devido a questionamentos em relação aos Fundamentos em Segurança, Meio Ambiente e Saúde (SMS). Todavia, a Radiografia Direta oferece tempos de aquisição equivalentes a segundos ou a poucos minutos, dependendo da aplicação. Esse fator torna-se favorável para trabalhos em campo, pois além de otimizar o tempo de inspeção, reduz a necessidade de grandes raios de isolamento.

Estudos complementares serão realizados no intuito de contribuir para a melhoria dos procedimentos descritos. É importante destacar que ainda não existem Indicadores de Qualidade de Imagem (IQI) para materiais compósitos. Por este motivo, é importante a busca de um padrão radiográfico para os defeitos já conhecidos e outros que possam existir.

\section{Agradecimentos}

Ao Conselho Nacional de Desenvolvimento Científico e Tecnológico (CNPq) pelo apoio financeiro e bolsas de estudo concedidas para este estudo (Processo 140937/2015-3)

\section{REFERÊNCIAS}

1 Neto FL, Pardini LC. Compósitos estruturais: ciência e tecnologia. São Paulo: Blucher; 2016.

2 Markovičová L, Zatkalíková V. Composite materials based on pa reinforced glass fibers. Materials Today: Proceedings. 2016; 3(4): 1056-1059.

3 Tan KT, Watanabe N, Iwahori Y. X-ray radiography and micro-computed tomography examination of damage characteristics in stitched composites subjected to impact loading. Composites: Part B. 2011; 42: 874-884.

4 Rique AM, Machado AC, Oliveira DF, Lopes RT, Lima I. X-ray imaging inspection of fiberglass reinforced by epoxy composite. Nuclear Instruments and Methods in Physics Research B. 2015; 349: 184-191.

5 Gholizadeh S. A review of non-destructive testing methods of composite materials. Procedia Structural Integrity 1. 2016;050-057. 
6 Lança L, Silva A. Digital radiography detectors e A technical overview: Part 1. Radiography. 2009; 15: 58-62.

7 Moreira EV, Rabello JM, Pereira MS, Lopes RT, Zacherpel U. Digital Radiography Using Digital Detector Arrays Fulfills Critical Applications for Offshore Pipelines. EURASIP Journal on Advances in Signal Processing. 2010; article number 894643.

8 ASTM E 2597, Standard Practice for Manufacturing Characterization of Digital Detector Arrays, 2007.

9 Azeredo SR, Oliveira DF, Gomes CS, Ferreira CG, Lopes RT. Otimização de dose em Radiografia Digital com DDA em juntas soldadas. In: 13ª Conferência sobre Tecnologia de Equipamentos; 2015; Recife, Brazil. 